\title{
Use of Intermittent Preventive Therapy for Malaria During Pregnancy and Development of Febrile IIIness Among Infants in Nigeria
}

\author{
Ngozi Anayochukwu-Ugwu ${ }^{1,2}$, Innocent Anayochukwu Ugwu ${ }^{3, *}$ \\ ${ }^{1}$ Nigeria Field Epidemiology and Laboratory Training Programme (NFELTP), Abuja, Nigeria \\ ${ }^{2}$ Department of Medical Laboratory, Federal Neuropsychiatric Hospital, Enugu, Nigeria \\ ${ }^{3}$ Department of Obstetrics \& Gynaecology, College of Medicine, Enugu State University of Science \& Technology (ESUT) and ESUT \\ Teaching Hospital, Parklane, Enugu, Nigeria
}

\section{Email address:}

ngozianayo2004@yahoo.com (N. Anayochukwu-Ugwu), innocent.ugwu@esut.edu.ng (I. A. Ugwu)

${ }^{*}$ Corresponding author

\section{To cite this article:}

Ngozi Anayochukwu-Ugwu, Innocent Anayochukwu Ugwu. Use of Intermittent Preventive Therapy for Malaria During Pregnancy and Development of Febrile Illness Among Infants in Nigeria. Journal of Gynecology and Obstetrics. Vol. 8, No. 1, 2019 , pp. 4-11. doi: $10.11648 /$ j.jgo.20200801.12

Received: November 19, 2019; Accepted: December 17, 2019; Published: January 6, 2020

\begin{abstract}
In most African countries, a good number of pregnant women make multiple antenatal visits providing a major opportunity for the prevention of malaria (associated with febrile illness) in infants through the use of intermittent preventive treatment in pregnancy (IPTp). This study assessed the association between maternal use of IPTp with sulphadoxine and pyrimethamine (SP) and the development of febrile illness in infants. This was a secondary data analysis of the 2013 Nigeria Demographic Health Survey (NDHS) data. Mother-child pairs where the mother was aged 15-49 years and the child was less than one year at the time of the survey were included. Variables such as the use of IPTp-SP and development of febrile illness as well as the socio-demographic and other control variable were analyzed. Chi-square testing and logistic regression were used to determine the association between the use of IPTp-SP and the development of febrile illness. Statistical analysis was done using SPSS version 21 and Statistical significance was set at $\mathrm{P}<0.05$. A total of 6,212 mother-child pairs were analyzed. Chi-square test showed that there was a significant association between the use of IPTp-SP and report of fever in infants. A higher proportion of mothers that used IPTp-SP (15.8\%) reported fever in their infants compared with those whose mother did not receive IPTp-SP $(11.6 \%)(\mathrm{P}<0.001)$. Logistic regression showed that mothers that used IPTp-SP were about one and half times more likely to report fever in their infants, before adjustment for confounding variables (OR $=1.46,95 \% \mathrm{CI}: 1.24-1.71, \mathrm{p}<0.001)$. Following adjustment, there was a weaker (though still significant) association between IPTp-SP use and fever in the infants (OR $=1.26,95 \%$ CI: $1.04-1.52, p=0.019)$. This study found a significant association between the use of IPTp-SP and mothers' report of febrile illness among infants in the two weeks before the survey. This requires further evidence to confirm but highlights the complex relationship between maternal drug exposure and long term susceptibility to illness in offspring.
\end{abstract}

Keywords: Intermittent Preventive Therapy, Sulphadoxine-pyrimethamine, Pregnancy, Febrile Illness, Malaria

\section{Introduction}

Malaria is a key public health problem both in the tropical and subtropical regions of the world and constitutes a major challenge to development and health, especially in subSaharan Africa, where children and pregnant women are at the greatest risk. Malaria in pregnancy is associated with many negative outcomes for mothers, fetuses, and infants [12]. Globally, approximately 125 million women living in malaria-endemic areas become pregnant annually; 30 million of whom live in sub-Saharan Africa [3]. In sub-Saharan Africa, malaria in pregnancy is responsible for $20 \%$ of all stillbirths [4]. Malaria in pregnancy thus contributes to a continuous cycle of illness, leading to an increased risk of 
low birth weight babies with increased susceptibility to ill health and death [5].

The symptoms as well as the complications of malaria in pregnancy vary with the acquired immunity state of the pregnant woman and thus with the intensity of malaria transmission [1]. While these settings are presented as two distinct epidemiologic conditions, in reality, the intensity of transmission and immunity in pregnant women occurs on a continuum, with potentially different conditions occurring within a country. In areas of unstable malaria transmission where adult pregnant women have no immunity, malaria in pregnancy can lead to severe consequences to both mother and fetuses, including death [1].

In areas of stable malaria transmission where there is immunity against malaria, pregnant women are relatively protected from severe consequences of malaria, however, due to sequestration of the malaria parasite in the placenta, fetal nutrition can be impaired leading to associated neonatal morbidities [1, 6, 7]. In order to prevent the adverse consequences of malaria in pregnancy in moderate and high transmission areas, the WHO recommends the use of intermittent preventive treatment in pregnancy (IPTp); a full preventive course of antimalarial medicine given to pregnant women at routine prenatal visits, regardless of whether the recipient is infected with malaria [7]. The WHO recommends IPTp with Sulphadoxine-Pyrimethamine (SP) for all pregnant women at each of the four scheduled antenatal care visits except in the first trimester [7]. Use of IPTp reduces maternal malaria episodes, maternal and fetal anemia, placental parasitemia, low birth weight, and neonatal mortality [8]. In most African countries over $70 \%$ of pregnant women make multiple antenatal clinic visits providing a major opportunity for the prevention of malaria through the use of IPTp [9-10]. However, in the last few years, it has been noted that there is a declining effort to scale-up IPTp in some African countries [7]; no country has achieved the $80 \%$ coverage target for 2 doses of IPTp.

More deaths are attributed to malaria in Nigeria than in any other nation of the world. Malaria accounts for $11 \%$ of maternal deaths and $20 \%$ of deaths among children less than 5 years of age in Nigeria [11]. Coverage of IPTp lags noticeably behind other malaria control measures with first and second dose coverage being $49 \%$ and $15 \% \%$ respectively [12]. Improving IPTp coverage requires more effective integration between maternal and child health $(\mathrm{MCH})$ programs and malaria control programs [5]. Understanding the relationship between use of IPT during pregnancy and development of febrile illness (which is the major symptom of malaria) among infants in Nigeria may provide evidence to enhance synergy between malaria control programs and maternal-child health services and thus contributes to the reduction of malaria-attributable infant mortality in Nigeria. The objective of this study was therefore to determine the association between maternal use of intermittent preventive treatment in pregnancy with sulphadoxine-pyrimethamine $\left(\mathrm{IPT}_{\mathrm{p}} \mathrm{SP}\right)$ and development of febrile illness among infants in Nigeria.

\section{Methodology}

\subsection{Study Design}

This was a descriptive epidemiological study that involved the analysis of secondary data obtained from the 2013 Nigeria Demographic and Health Survey (NDHS).

\subsection{Study Setting}

The study was conducted in Nigeria, the most populous country in Africa with a population of $177,155,754$ [13]. Nigeria is made of 36 states. The states are divided into 774 local government areas (LGA's) which are split to localities. There are up to 374 various ethnic groups; the largest are the Hausa and Fulani mainly found in the northern part, the Igbo in the eastern part, and the Yoruba in the south-western part of the country. The Hausa and Fulani are predominantly Muslim, the Igbo mainly Christian, and there is a balance in the two religions among the Yoruba. Regional differences exist in service delivery as well as status and resource availability in the health sector, with more health care services in the south when compared with the northern region of the country [12].

\subsection{Inclusion-exclusion Criteria/Sample Size}

Mother-Child pairs where the mother was aged 15-49 years and the child was less than one year at the time of the survey were included. Women who had no birth in the past five years were excluded. A total of 38,948 women aged 1549 years were included in the 2013 NDHS, of whom 6,212 met the inclusion and exclusion criteria.

\subsection{Ethics}

Individual-level data from the 2013 Nigeria Demographic Survey was made available by request from ICF macro USA. Informed consent was provided for the initial data collection; because this was a secondary analysis, no further consent was needed and local ethical approval was not sought.

\subsection{Data Source}

The data was derived from the women's questionnaire of the 2013 Nigeria Demographic and Health Survey (NDHS). It provided recent information on 'health indicators' including use of IPTp-SP, health of infants as well as other demographic characteristics of women of reproductive age (15-49 years).

\subsection{Definitions of Variables}

The development of febrile illness in the infant was the dependent variable. This is defined as a fever within the last two weeks in infants less than or equal to 12 months of age. The independent variables include the use of IPTp-SP to prevent malaria during pregnancy. This is defined as the use of any of the following drugs: Sulphadoxine-pyrimethamine (SP), fansidar, Amalar or Maloxine during pregnancy. For those who took the drugs, other variables include: the number 
of times the drug was taken during pregnancy as well as place of access of the drug (was the drug given during an antenatal care visit or visit to other health facilities?) Other control variables include mother's age at last delivery, educational status, wealth quintile, place of residence (urban or rural), region of residence, marital status, religion and number of children.

\subsection{Description of Primary Survey/Sampling Approach/Sampling Frame}

The sampling frame of the primary survey was the list of enumeration areas (EA's) from the 2006 Nigerian national population census. The sample was got using a stratified three-stage cluster design. Stratification was obtained by splitting all states in the nation into urban and rural areas. During the first stage, a total of 839 different localities were selected using independent selection in every stratum with probability proportional to size. In the second stage, enumeration areas were chosen randomly from the localities with the selection of greater than one enumeration area from some bigger localities. A total of 904 clusters (EA's), 372 in the urban and 532 in the rural area were selected. Finally, during the third stage of selection, 45 households were selected from each urban and rural cluster by equal probability systematic sampling using updated households list from stage 2 . A total of 40,680 households were selected and used for the survey. All women age 15- 49 years who were permanent residents or who slept in the house on the night before the interviews in the selected household were qualified for interviews. In total, 38,948 were interviewed. Interviewers were trained and the questionnaires were pretested by the trained interviewers. The questionnaires were designed in English but were translated to the 3 major indigenous Nigerian languages. Fieldwork and data collection was carried out in four months. The response rate was $98 \%$. Data entry was done during fieldwork to allow for easy identification and correction of inconsistencies and errors [12].

\subsection{Data Collection/Analysis Plan}

This analysis used data collected in the women's questionnaire, which contained questions relating to maternal and child health, fertility, etc. Statistical analysis was done using SPSS version 21. For this analysis, only those with responses for the variables of interest were included in crosstabulation (chi-square test) and logistic regression; all cases with missing data were also excluded using the complete case analysis method [14]. Also to address the differences in the probability of selection and produce an appropriate representation, weighting was applied in the tabulations [15]. The weight case option in SPSS was used for all the crosstabulations and logistic regression analyses.

Descriptive statistics were used to show the sociodemographic characteristics of the participants. Frequency tables were used to show the percentage of sociodemographic characteristics as well as the use of IPTp. The
Chi-square test was used to test for the association between the independent variables (socio-demographic characteristics, IPTp use) and dependent variables (development of febrile illness in infants). Multiple logistic regression models were used to test the relationship between the dependent and the independent variables as well as to address confounding. Data were considered statistically significant with $\mathrm{p}<0.05$.

\section{Result}

\subsection{Descriptive Statistics}

\subsubsection{Frequency Distribution of Mothers' and Childs' Sociodemographic Characteristics}

Out of the total of 38,948 women aged 15-49 years included in the data set, the analysis included a total of 6,212 mother-child pairs where the child was less than one year at the time of the survey. The highest proportion of the women was aged 25-29 years (28.2\%) followed by those aged 20-24 years $(23.5 \%)$ and $30-34$ years $(20.4 \%)$ (Table 1$)$. About twothirds $(66.2 \%)$ were from rural areas. Over half of the respondents $(57.8 \%)$ were Muslims while almost half $(44.3 \%)$ had no formal education. The majority of the women were currently in a union $(95.7 \%)$. Except for infants less than 1 month, there were similar numbers of infants across each month (Table 2).

Table 1. Frequency distribution of mothers' socio-demographic characteristics.

\begin{tabular}{lll}
\hline Variable & Frequency & \% \\
\hline Age (years) & & \\
$15-19$ & 548 & 8.8 \\
$20-24$ & 1462 & 23.5 \\
$25-29$ & 1754 & 28.2 \\
$30-34$ & 1265 & 20.4 \\
$35-39$ & 783 & 12.6 \\
$40-44$ & 306 & 4.9 \\
$45-49$ & 94 & 1.5 \\
Region & & \\
Northcentral & 898 & 14.5 \\
Northeast & 1258 & 20.3 \\
Northwest & 1922 & 30.9 \\
Southeast & 574 & 9.2 \\
South-south & 755 & 12.2 \\
Southwest & 805 & 13.0 \\
Place of residence & & \\
Urban & 2097 & 33.8 \\
Rural & 4115 & 66.2 \\
Highest educational level & & \\
None & 2755 & 44.3 \\
Primary & 1184 & 19.1 \\
Secondary & 1865 & 30.0 \\
Tertiary & 408 & 6.6 \\
Wealth index & & \\
Poorest & 1337 & 21.5 \\
Poorer & 1274 & 22.8 \\
Middle & 1176 & 18.9 \\
Richer & 1011 & 16.3 \\
Richest & & \\
Religion & & \\
Catholic & & \\
Other Christian & & \\
Islam & & \\
\hline & & \\
\hline
\end{tabular}




\begin{tabular}{lll}
\hline Variable & Frequency & $\mathbf{\%}$ \\
\hline Traditionalist & 45 & 0.7 \\
Other & 4 & 0.1 \\
Missing & 34 & 0.5 \\
Number of children & & \\
$1-2$ & 2595 & 41.8 \\
$3-4$ & 1908 & 30.7 \\
$5+$ & 1709 & 27.5 \\
Marital status & & \\
Never in union & 170 & 2.7 \\
Currently in union & 5944 & 95.7 \\
Formerly married & 98 & 1.6 \\
\hline
\end{tabular}

Table 2. Frequency distribution of Childs' socio-demographic characteristics.

\begin{tabular}{lll}
\hline Variable & Frequency & Percentage (\%) \\
\hline Age of child (months) & & \\
0 & 277 & 4.5 \\
1 & 537 & 8.6 \\
2 & 526 & 8.5 \\
3 & 553 & 8.9 \\
4 & 572 & 9.2 \\
5 & 531 & 8.5 \\
6 & 566 & 9.1 \\
7 & 547 & 8.8 \\
8 & 539 & 8.7 \\
9 & 561 & 9.0 \\
10 & 554 & 8.9 \\
11 & 449 & 7.2 \\
Gender of child & & \\
Male & 3079 & 49.6 \\
Female & 3133 & 50.4 \\
\hline
\end{tabular}

\subsubsection{Proportion of Children Under 1 Year with Fever Two Weeks Before the Interview}

Only $12.6 \%$ of children less than one year were reported to have had a fever in the two weeks before the interview (Table 3).

Table 3. Proportion of children aged less than 12 months that had a fever 2 weeks before the interview.

\begin{tabular}{lll}
\hline Variable & Frequency & $\mathbf{\%}$ \\
\hline Fever 2 weeks before interview & & \\
Yes & 780 & 12.6 \\
No & 5406 & 87.0 \\
Don't know & 2 & 0.0 \\
Missing & 24 & 0.4 \\
\hline
\end{tabular}

\subsubsection{Antenatal Care Attendance and IPT Use}

Although about $51 \%$ of women reported having made at least 4 ANC visits, nearly one-third reported never visited $\mathrm{ANC}$, and only $27 \%$ reported ever having used IPTp-SP (Table 4). Of this number, 39\% used one dose, 36.9\% used two doses, and less than a quarter $(22.9 \%)$ used three or more doses. More than three-quarters of women reporting IPT use got the drugs during their ANC visit.

Table 4. ANC attendance and IPT use in last pregnancy by mothers of children aged less than 12 months.

\begin{tabular}{lll}
\hline Variable & Frequency & \% \\
\hline ANC visits & & \\
None & 1922 & 30.9 \\
$1-3$ & 889 & 14.3 \\
$4+$ & 3163 & 50.9 \\
Don't know/No response & 238 & 3.8 \\
Used IPT last pregnancy & & \\
Yes & 1658 & 26.7 \\
No & 4371 & 70.4 \\
Don't know & 78 & 1.3 \\
Missing & 105 & 1.6 \\
Number of IPT used $(\mathrm{n}=1658)$ & & \\
1 & 646 & 39.0 \\
2 & 611 & 36.9 \\
$3+$ & 379 & 22.9 \\
Missing & 22 & 1.3 \\
Source of IPT $(\mathrm{n}=1658)$ & & \\
ANC visit & 1352 & 81.5 \\
Other facilities & 59 & 3.6 \\
Other sources & 16 & 1.0 \\
Missing & 231 & 13.9 \\
\hline
\end{tabular}

\subsection{Bivariate Result}

\subsubsection{Association Between Mothers' IP Use in Pregnancy and Fever in the Infant}

There was a significant association between the use of IPTp-SP and fever in infants (Table 5). A higher proportion of mothers that reported IPT use in pregnancy $(15.8 \%)$ reported fever in their infants compared with those whose mother did not receive IPTp $(11.6 \%)(\mathrm{P}<0.001)$. There was no significant association between the number of IPT doses and fever $(\mathrm{P}=0.781)$.

Table 5. Association between mothers' IPTP-SP use and fever 2 weeks before the interview.

\begin{tabular}{|c|c|c|c|c|c|}
\hline \multirow{2}{*}{ Variable } & \multicolumn{5}{|c|}{ Fever 2 weeks before interview } \\
\hline & Yes $(\%)$ & No $(\%)$ & Total & Chi square & P value \\
\hline \multicolumn{6}{|l|}{ IPT use } \\
\hline Yes & $262(15.8)$ & $1391(84.2)$ & 1653 & \multirow{2}{*}{19.85} & \multirow{2}{*}{$<0.001$} \\
\hline No & $503(11.6)$ & $3849(88.4)$ & 4352 & & \\
\hline \multicolumn{6}{|c|}{ Number of IPT doses } \\
\hline 1 & $102(15.8)$ & $543(84.2)$ & 645 & \multirow{3}{*}{0.49} & \multirow{3}{*}{0.781} \\
\hline 2 & $92(15.1)$ & $518(84.9)$ & 610 & & \\
\hline $3+$ & $63(16.8)$ & $313(83.2)$ & 376 & & \\
\hline
\end{tabular}

\subsubsection{Association Between Mothers' Characteristics and Fever in the Infant}

infant and regions, with fever most commonly reported in the North East and South East compared to other regions. Fever in the infants was significantly higher among older infants 
(6-11 months) and women with fewer than 4 ANC visits and women currently in union (Table 6). was lowest among women in the richest wealth index and

Table 6. Association between mothers/ infants characteristics and developments offever 2 weeks before the interview.

\begin{tabular}{|c|c|c|c|c|c|}
\hline \multirow{2}{*}{ Variable } & \multicolumn{5}{|c|}{ Fever 2 weeks before interview } \\
\hline & Yes $(\%)$ & No (\%) & Total & P value & Chi square \\
\hline \multicolumn{6}{|c|}{ Mother's Age (years) } \\
\hline $15-19$ & $77(14.3)$ & $463(85.7)$ & 540 & \multirow{4}{*}{0.474} & \multirow{3}{*}{1.49} \\
\hline $20-34$ & $558(12.5)$ & 3909 (87.5) & 4467 & & \\
\hline $35-49$ & $145(12.3)$ & $1034(87.7)$ & 1179 & & \\
\hline \multicolumn{5}{|l|}{ Region } & \\
\hline Northcentral & $54(6.0)$ & $839(94.0)$ & 893 & \multirow{6}{*}{$<0.001$} & \multirow{6}{*}{218.03} \\
\hline Northeast & $273(21.8)$ & $980(78.2)$ & 1253 & & \\
\hline Northwest & $203(10.6)$ & $1711(89.4)$ & 1914 & & \\
\hline Southeast & $117(20.5)$ & $454(79.5)$ & 571 & & \\
\hline South-south & $97(12.9)$ & $655(87.1)$ & 752 & & \\
\hline Southwest & $36(4.5)$ & $767(95.5)$ & 803 & & \\
\hline \multicolumn{6}{|l|}{ Place of residence } \\
\hline Urban & $254(12.1)$ & $1838(87.9)$ & 2092 & \multirow{2}{*}{0.428} & \multirow{2}{*}{0.63} \\
\hline Rural & $526(12.8)$ & $3568(87.2)$ & 4094 & & \\
\hline \multicolumn{6}{|c|}{ Mother's Highest educational level } \\
\hline None & $345(12.6)$ & $2393(87.4)$ & 2738 & \multirow{5}{*}{0.277} & \multirow{4}{*}{3.86} \\
\hline Primary & $154(13.1)$ & $1026(86.9)$ & 1180 & & \\
\hline Secondary & $242(13.0)$ & $1619(87.0)$ & 1861 & & \\
\hline Tertiary & $39(9.6)$ & $368(90.4)$ & 407 & & \\
\hline \multicolumn{5}{|l|}{ Wealth index } & \\
\hline Poorest & $179(13.4)$ & $1155(86.6)$ & 1334 & \multirow{5}{*}{0.003} & \multirow{5}{*}{16.07} \\
\hline Poorer & $201(14.3)$ & $1203(85.7)$ & 1404 & & \\
\hline Middle & $172(13.5)$ & $1099(86.5)$ & 1271 & & \\
\hline Richer & $132(11.3)$ & $1036(88.7)$ & 1168 & & \\
\hline Richest & $96(9.5)$ & $913(90.5)$ & 1009 & & \\
\hline \multicolumn{6}{|l|}{ Religion } \\
\hline Christianity & $300(11.8)$ & $2232(88.2)$ & 2532 & \multirow{2}{*}{0.164} & \multirow{2}{*}{1.94} \\
\hline Islam & $466(13.0)$ & $3106(87.0)$ & 3572 & & \\
\hline \multicolumn{6}{|l|}{ Number of children } \\
\hline $1-2$ & $322(12.5)$ & $2257(87.5)$ & 2579 & \multirow{3}{*}{0.716} & \\
\hline $3-4$ & $234(12.3)$ & $1670(87.7)$ & 1904 & & 0.67 \\
\hline $5+$ & $224(13.2)$ & $1479(86.8)$ & 1703 & & \\
\hline Marital status & & & & & \\
\hline Never in union & $30(17.8)$ & $139(82.2)$ & 169 & & \\
\hline Currently in union & $733(12.4)$ & $5186(87.6)$ & 5919 & 0.042 & 6.33 \\
\hline Formerly married & $17(17.3)$ & $81(82.7)$ & 98 & & \\
\hline ANC visits & & & & & \\
\hline None & $192(10.0)$ & $1720(90.0)$ & 1912 & & \\
\hline $1-3$ & $148(16.7)$ & $737(83.3)$ & 885 & $<0.001$ & 25.57 \\
\hline $4+$ & $412(13.1)$ & $2741(86.9)$ & 3153 & & \\
\hline Age of child (month & & & & & \\
\hline Less than 1 & $6(2.2)$ & $269(97.8)$ & 275 & & \\
\hline $1-5$ & $215(7.9)$ & $2492(92.1)$ & 2707 & $<0.001$ & 148.69 \\
\hline $6-11$ & $559(17.4)$ & $2645(82.6)$ & 3204 & & \\
\hline Gender of child & & & & & \\
\hline Male & $408(13.3)$ & $2657(86.7)$ & 3065 & ก 000 & 77 \\
\hline Female & $372(11.9)$ & $2749(88.1)$ & 3121 & 0.099 & 2.12 \\
\hline
\end{tabular}

\subsection{Logistic Regression}

\subsubsection{Simple/Multiple Logistic Regression of Mothers' IPT}

Table 7. Crude and Adjusted Odds ratios assessing the relationship between mothers IPTp-SP use and report of fever in the infants in the 2 weeks before the interview.

\begin{tabular}{|c|c|c|c|c|c|c|}
\hline \multirow{2}{*}{$\begin{array}{l}\text { Variable } \\
\text { IPT use }\end{array}$} & \multicolumn{3}{|c|}{ Simple logistic regression } & \multicolumn{3}{|c|}{ Multiple logistic regression } \\
\hline & Crude Odds ratio & 95\% confidence interval & P-value & Adjusted* Odds ratio & $\mathbf{9 5 \%}$ confidence interval & P-value \\
\hline $\begin{array}{l}\text { Yes } \\
\text { No (ref) }\end{array}$ & $\begin{array}{l}1.46 \\
1 \\
\end{array}$ & $1.24-1.71$ & $<0.001$ & $\begin{array}{l}1.26 \\
1 \\
\end{array}$ & $1.04-1.52$ & 0.019 \\
\hline
\end{tabular}

*Adjusted for mother and child's socio-demographic characteristics, and antenatal visits as shown in Table 7. 
Mothers that used IPTp-SP were about one and half times more likely to report fever in their infants, before adjustment for confounding variables $(\mathrm{OR}=1.46,95 \% \mathrm{CI}: 1.24-1.71$, $\mathrm{p}<0.001$ ). Following adjustment, there was a weaker (though still significant) association between IPTp-SP use and fever in the infants $(\mathrm{OR}=1.26,95 \% \mathrm{CI}: 1.04-1.52, \mathrm{p}=0.019)$ (Table 7).

\subsubsection{Multiple Logistic Regressions of Mothers' and Infants' Characteristics on Fever in Infants}

The odds ratio from multiple logistic regression of IPTpSP use on fever in the infants, adjusted for mothers' sociodemographic status, antenatal care, and infant characteristics are shown in table 8. Respondents from the Southeast were most likely to report a fever in their children, while respondents from the Southwest were the least likely to report a fever in their children. Poorer women were significantly more likely to report fever in their infant in the two weeks before the interview. The odds of fever were significantly higher among older children, Muslims, and women that had attended more ANC visits.

Table 8. Multiple logistic regression of having fever 2 weeks before the interview and mothers' socio-demographic characteristics.

\begin{tabular}{|c|c|c|c|}
\hline Variable & Odds ratio (OR) & 95\% CI OR & P value \\
\hline \multicolumn{4}{|l|}{ IPT use } \\
\hline Yes & 1.26 & \multirow{3}{*}{$1.04-1.52$} & \multirow{3}{*}{0.019} \\
\hline No (ref) & 1 & & \\
\hline \multicolumn{2}{|l|}{ Age (years) } & & \\
\hline $15-19$ & 1.07 & \multirow{3}{*}{$0.82-1.33$} & 0.736 \\
\hline $20-34$ & 1.04 & & \multirow{2}{*}{0.726} \\
\hline 35-49 (ref) & 1 & & \\
\hline \multicolumn{4}{|l|}{ Region } \\
\hline Northcentral & 1.23 & $0.78-1.94$ & 0.375 \\
\hline Northeast & 4.88 & $3.22-7.39$ & $<0.001$ \\
\hline Northwest & 2.04 & $1.32-3.15$ & 0.001 \\
\hline Southeast & 5.49 & $3.59-8.39$ & $<0.001$ \\
\hline South-south & 3.25 & $2.10-5.04$ & $<0001$ \\
\hline Southwest (ref) & 1 & & -0.001 \\
\hline \multicolumn{4}{|l|}{ Wealth index } \\
\hline Poorest & 1.56 & $1.05-2.33$ & 0.030 \\
\hline Poorer & 1.64 & $1.13-2.38$ & 0.010 \\
\hline Middle & 1.48 & $1.06-2.07$ & 0.022 \\
\hline Richer & 1.07 & $0.78-1.47$ & 0668 \\
\hline Richest (ref) & 1 & & 0.000 \\
\hline \multicolumn{4}{|l|}{ Religion } \\
\hline Christianity & 0.69 & $0.52-0.92$ & \multirow{2}{*}{0.012} \\
\hline Islam (ref) & 1 & & \\
\hline \multicolumn{4}{|l|}{ Number of children } \\
\hline $1-2$ & 0.90 & $0.70-1.15$ & 0.388 \\
\hline $3-4$ & 0.98 & $0.78-1.24$ & \multirow{2}{*}{0.862} \\
\hline $5+(\mathrm{ref})$ & 1 & & \\
\hline \multicolumn{4}{|l|}{ Marital status } \\
\hline Never in union (ref) & 1 & & 0.188 \\
\hline Currently in union & 0.73 & $0.46-1.16$ & \multirow{2}{*}{0.660} \\
\hline Formerly married & 0.85 & $0.42-1.73$ & \\
\hline \multicolumn{4}{|l|}{ Age of child (months) } \\
\hline Less than 1 (ref) & 1 & & \\
\hline $1-5$ & 4.40 & $1.79-10.86$ & 0.001 \\
\hline $6-11$ & 11.52 & $4.70-28.22$ & $<0.001$ \\
\hline \multicolumn{4}{|l|}{ Gender of child } \\
\hline Male & 1.12 & $0.96-1.32$ & \\
\hline Female & 1 & & 0.151 \\
\hline ANC visits & & & \\
\hline
\end{tabular}

\begin{tabular}{|c|c|c|c|}
\hline Variable & Odds ratio (OR) & 95\% CI OR & P value \\
\hline None (ref) & 1 & & \\
\hline $1-3$ & 1.55 & $1.19-2.02$ & 0.001 \\
\hline $4+$ & 1.40 & $1.10-1.78$ & 0.007 \\
\hline
\end{tabular}

\section{Discussion}

Malaria which often presents as a febrile illness accounts for about 214 million cases and 438,000 deaths in 2015 and most of the disease burden occurs in Sub-saharan Africa [16]. This descriptive study examined the relationship between the use of intermittent preventive therapy for malaria during pregnancy (IPTp) and the development of febrile illness among infants in Nigeria. There was a significant association between IPTp-SP use and fever in infants. A higher proportion of mothers that had used IPT in pregnancy reported fever in their infants in the two weeks before the survey compared with mothers who did not receive IPTp during pregnancy. This finding is consistent with that of other studies in similar settings. In a birth cohort study, IPTp-SP was associated with a higher risk of 'malaria outcome' (febrile illness) among infants [17]. It has also been previously noted that in communities where there is widespread resistance to (SP), the use of IPTp-SP did not prevent placental malaria sequestration but rather was associated with increased malaria infections $[18,19]$.

Use of IPTp-SP could exacerbate placental malaria infection which in turn predicts increased risk of malaria parasitemia as well as the occurrence of overt clinical malaria in offspring [20-22]. Evidence has suggested that IPTp- SP use in humans could decrease anti-malaria antibodies in mothers though this is modified by HIV status and parity [23, 24]. However, it also possible that the use of IPTp-SP may decrease the variety of the 'maternal anti-malaria antibodies' resulting in increased infant vulnerability to diverse malaria parasite populations [17]. Treating malaria in mice before pregnancy has been linked with reduced anti-malaria antibodies, increased malaria parasitemia, as well as increased morbidity and mortality in their pups after infection with malaria [25]. Similarly, it was demonstrated that the use of IPTp during pregnancy was unexpectedly associated with low cord blood levels and a higher risk of fetal anemia in Tanzania [19]. Fetal anemia plays a large role in predicting infant mortality and morbidity. Anemia in fetuses predisposes to anemia in infancy [26] which may be implicated in childhood diseases like respiratory infection, diarrhea or malaria (that may be associated with febrile illness) and increased occurrence of persistent diseases as well as an increased collective incidence of disease morbidity [27]. Decreasing compliance, growing resistance to SP treatment as well as other anti-malarial medicines draws attention to the complex nature of IPTp-SP treatment [29] The suggested alternative to IPTp drug treatment in places with considerable resistance against SP is 'the Intermittent Screening and Treatment (IST) [29]. This regimen involves using a rapid diagnostic test (RDT) to screen for malaria infection at a scheduled antenatal care visitation and then subsequently 
treat women who are positive with very effective antimalarial treatment [30].

However, other groups have failed to replicate the findings by Harrington et al. Use of IPTp-SP did not exacerbate pregnancy-associated morbidities despite the increasing prevalence of SP resistant strains of Plasmodium falciparum and it was noted that even in the presence of appreciable resistance, (SP) could still be taken as IPTp [28]. However, the need to find an alternative drug for prevention was reiterated. In a prospective randomized, placebo-controlled trial, it was noted that IPTp with SP in Mozambican pregnant women also led to a significant reduction in neonatal mortality in their offspring that were followed up to 12 months [8]. Decreasing compliance, growing resistance to SP treatment as well as other antimalarial medicines draws attention to the complex nature of IPTp-SP treatment [29].

\subsection{Study Strengths}

The major strength of this study was that the data was representative of Nigeria. The large sample size used for this analysis, the high rate of response and participation and standard method of collection of data all ensured reliability and representativeness of data. The span of the data collected allowed a deep analysis that went beyond counting prevalence but enabled the determination of the relationship between the use of IPTp-SP and the occurrence of febrile illness in infants. Therefore the result from this study reflects the use of IPTp-SP and the development of febrile illness among infants in the nation.

\subsection{Study Limitations}

This data was derived from a cross-sectional survey, and therefore causality cannot be determined. The use of (IPTp) was significantly associated with mother's reporting a febrile illness in the two weeks preceding the survey. However, it was not possible to conclude a cause-effect relationship. It was assumed that the major cause of febrile illness especially Nigeria is malaria. However, other conditions may cause fever, and these were not differentiated by our study. There is also the possibility that the association we found might be a result of increasing exposure to malaria among the children of mothers that used IPTp as this was not a randomized controlled trial. This may not necessarily be the case as the use of IPTp was national policy and distribution was done without preference to malaria disease prevalence.

\section{Conclusion}

In conclusion, this study found a significant association between the use of intermittent preventive therapy for malaria during pregnancy (IPTp) using Sulphadoxinepyrimethamine (SP) and mothers' report of febrile illness among infants in the two weeks before the survey. These findings, along with that of other studies as discussed above, raised concerns of potential harm following the use of
Sulphadoxine- pyrimethamine and may imply that the continued use of a possibly failing drug could worsen instead of alleviating disease and infections in certain individuals. It also highlighted the complex relationship between maternal drug exposure and long term susceptibility to illness in offspring. Future research should aim at a comparative study of IPTp-SP and Intermittent screening and treatment (IST) during pregnancy or in the alternative, trying differing drugs to find a better alternative to use for the global malaria control programs.

\section{Funding}

This work was supported by Task Force For Global Health Inc., USA/ Training Programs in Epidemiology and Public Health Interventions Network (DocuSign Envelope ID: E0648FF5-50B2-4FFE-9686-863B6B00F7A1).

\section{Acknowledgements}

The authors wish to express their appreciation to MEASURE DHS and the ICF macro USA for granting access to 2013 Nigeria Demographic and Health Survey data.

\section{References}

[1] Desai, M. I., Kuile, F. O, Nosten, F., McGready, R., Asamoa, K. Brabin, B. et al., (2007). Epidemiology and burden of malaria in pregnancy. Lancet of Infectious Disease. 7 (2), pp. 93-104.

[2] World Health Organization (2015) 'Malaria in pregnant women' [Online] Available at http://www.who.int/malaria/areas/high_risk_groups/pregnancy /en/ (Accessed 6 April 2015).

[3] Dellicour, S. Andrew J. T., Carlos A. G., Robert W. S., Feiko O., et al., (2010). Quantifying the Number of Pregnancies at Risk of Malaria in 2007: A Demographic Study. PLoS Med, 7 (1) e1000221. https://doi.org/10.1371/journal.pmed.1000221.

[4] Lawn, E. J., Blencowe, H2, Waiswa P3, Amouzou A4, Mathers C5, Hogan D5et al.,(2016). Stillbirths: rates, risk factors, and acceleration towards 2030. The Lancet, 387 No 10018, pp. 587-603.

[5] Robalo et al, (n. d) 'Opportunities for Africa's newborns' [Online] Available at http://www.who.int/pmnch/media/publications/oanfullreport.p df (Accessed 27 September 2015).

[6] Mulamba, M. and Mash, B., (2010). Evaluation of malaria prevention strategies during pregnancy in Ndola, Zambia. African Journal of Primary Health Care \& Family Medicine, 2 (1): pp. 1-5.

[7] World Health Organization, (2015). 'Intermittent preventive treatment in pregnancy (IPTp)' [Online] Available at http://www.who.int/malaria/areas/preventive_therapies/pregna ncy. (Accessed 10 April 2016). 
[8] Menendez, C., Bardaj1, A., Sigauque, B., Sanz, S., Aponte, J. J., Mabunda, S., et al. (2010). Malaria Prevention with IPTp during Pregnancy Reduces Neonatal Mortality. PLoS ONE, 5 (2), 1-6. doi: 10.1371.

[9] National Population Commission (NPC) [Nigeria] and ICF International. (2014). Nigeria Demographic and Health Survey 2013. Abuja, Nigeria, and Rockville, Maryland, USA: NPC and ICF International.

[10] Ntirushwa, D. (2010). A Strategic Framework for Malaria Prevention and Control during Pregnancy in the Africa Region. [Online] Available at https://www.gfmer.ch/SRHCourse-2010/assignments/pdf/Malaria-pregnancy-Ntirushwa2010.pdf (Accessed 31 November 2019).

[11] Nigeria Malaria Fact Sheet, (2011).Malaria in Nigeria [Online] Available at: http://photos.state.gov/libraries/nigeria/231771/Public/Decem ber-MalariaFactSheet2.pdf (Accessed 27 September 2015).

[12] National Population Commission (NPC) [Nigeria] and ICF Macro. (2009). Nigeria Demographic and Health Survey 2008. Abuja, Nigeria: National Population Commission and ICF Macro.

[13] Central Intelligence Agency, (2015). Central Intelligence Agency (CIA). [Online] Available at: $\mathrm{http}: / /$ www.theodora.com/wfbcurrent/nigeria/nigeria people.h tml (Accessed 9 April 2016).

[14] Graham, J. W. (2009) 'Missing data analysis: making it work in the real world' Annu. Rev. Psychol. 60: pp. 549-576 [Online] Available

at http://www.annualreviews.org.ezproxy.liv.ac.uk/doi/pdf/10.11 46/annurev.psych.58.110405.085530 (Accessed 22 September 2015).

[15] DHS Programme, (n. d) Sapling Weights. http://www.dhsprogram.com/data/Data-Quality-andUse.cfm\#CP_JUMP_5188 (Accessed 10 April 2016).

[16] World Health Organization (2016) 'Malaria and disease burden' [Online] Available at http://www.who.int/mediacentre/factsheets/fs094/en/. (Accessed 30 September 2016).

[17] Harrington, W., Morrison R, Fried M, D. P. (2013). Intermittent Preventive Treatment in Pregnant Women Is Associated with Increased Risk of Severe Malaria in Their Offspring. PLoS ONE, 8 (2).

[18] Harrington, W. et al., (2009). Competitive facilitation of drugresistant Plasmodium falciparum malaria parasites in pregnant women who receive preventive treatment Proc Natl Acad Sci U SA., 106, pp. 9027-9032.

[19] Harrington, W. et al., (2011). "Intermittent Treatment to Prevent Pregnancy Malaria Does Not Confer Benefit in an Area of Widespread Drug Resistance". Clinical Infectious Diseases. 53 (3), pp. 224-230.

[20] Mutabingwa, T. K. I., Bolla, M. C, Li J. L., Domingo, G. J., Li X., Fried M, et al., (2005). Maternal malaria and gravidity interact to modify infant susceptibility to malaria. PLoS Med, 2: e407.

[21] Le Hesran, J. M., Michel Cot, Personne, P., Fievet, N., Dubois, B., Beyeme, M. et al (1997). Maternal placental infection with Plasmodium falciparum and malaria morbidity during the first 2 years of life. American Journal of Epidemiology, Volume 146, (10) pp. 826-831.

[22] Schwarz, N. G., Adegnika, A. A., Breitling, L. P., Gabor, J., Agnandji, S. T and Newman, R. D. (2008). Placental malaria increases malaria risk in the first 30 months of life. Clin Infect Dis, 47, pp. 1017-1025.

[23] Serra-Casas, E., Menéndez, C., Bardají, A., Quintó, A., Dobaño, C., Sigauque, B. et al. (2010). The effect of intermittent preventive treatment during pregnancy on malarial antibodies depends on HIV status and is not associated with poor delivery outcomes. Journal of Infectious Diseases, 201 (1) pp 123-131.

[24] Aitken, E. H., Mbewe, B., Luntamo, M., Kulmala, T., Beeson, J. G and Ashorn, P (2012). Antibody to P. falciparum in pregnancy varies with intermittent preventive treatment regime and bed net use. PLoS ONE 7 (1): e29874. https://doi.org/10.1371/journal.pone.0029874.

[25] Vincent Staszewski, V, Reece, S. E., O'Donnell, A. O and Cunningham, E. J. A. (2012). Drug treatment of malaria infections can reduce levels of protection transferred to offspring via maternal immunity. Proc Biol Sci, 279 (1737) pp. 2487-2496.

[26] le Cessie, S., Verhoeff, F., Mengistie, G., Kazembe, P., Broadhead, R and BrabinLe, B. (2002). Changes in haemoglobin levels in infants in Malawi: effect of low birth weight and fetal anaemia. Arch Dis Child Fetal Neonatal Ed. 86 (3) pp 182-187.

[27] Kalanda, B., Verhoeff. F., le Cessie, S and Brabin J. Low birth weight and fetal anaemia as risk factors for infant morbidity in rural Malawi. Malawi Med J. 2009 Jun; 21 (2): 69-74.

[28] Desai, M., Gutman, J., Taylor, S. M., Wiegand, R. E., Khairallah, C., Kayentao et al, (2016). Impact of SulfadoxinePyrimethamine Resistance on Effectiveness of Intermittent Preventive Therapy for Malaria in Pregnancy at Clearing Infections and Preventing Low Birth Weight. Clin Infect Dis. Feb 1; 62 (3): 323-333. doi: 10.1093/cid/civ881.

[29] Moya-Alvarez, V. Abellana, R. and Cot, M. (2014). Pregnancy-associated malaria and malaria in infants: an old problem with present consequences. Malaria Journal. 13, Article number: 271. [Online] Available at: https://malariajournal.biomedcentral.com/articles/10.1186/147 5-2875-13-271 Accessed 6 November 2019.

[30] Tagbor, H., Bruce, J., Agbo, M., Greenwood, B. and Chandramohan, D (2010). Intermittent screening and treatment versus intermittent preventive treatment of malaria in pregnancy: a randomized controlled non-inferiority trial. PLoS ONE, 5: e14425. [Online Available at https://journals.plos.org/plosone/article?id=10.1371/journal.po ne.0014425 Accessed 6 November 2019. 